\title{
EXPERIENCIAS
}

\section{LA EXPERIENCIA EN EL PROGRAMA DE ADAPTACIÓN CURRICULAR EN GRUPO EN EL IES «MATILDE SALVADOR» DE CASTELLÓN DE LA PLANA DURANTE LOS CURSOS 99/00 Y 00/01.}

\author{
AN EXPERIENCE WITH THE «PROGRAMA DE ADAPTACIÓN CURRICULAR \\ EN GRUPO» AT THE IES «MATILDE SALVADOR» IN CASTELLON DE LA \\ PLANA, DURING THE YEARS 99/00 AND 00/01
}

José Juan Sidro*

IES Matilde Salvador

\begin{abstract}
RESUMEN
El desarrollo de la LOGSE (1990) en el campo de la atención a la diversidad en los Institutos de Educación Secundaria (IES) tiene como marco de referencia en la Comunidad Valenciana la Orden de Atención a la Diversidad de 29 de junio de 1999. En ella se establece el Plan de Actuación para la atención a la diversidad del alumnado con medidas de carácter general junto a medidas específicas.

En este artículo pretendemos hacer un análisis y valoración detallada de lo que ha supuesto poner en marcha en nuestro instituto la medida mas específica de atención a la diversidad, conocida como Programa de Adaptación Curricular en Grupo y al mismo tiempo extraer una serie de conclusiones derivadas de la experiencia en relación con el alumnado, el profesorado, los resultados, los servicios de orientación y apoyo, el currículo, la programación, los materiales y recursos y, la incidencia en el centro.
\end{abstract}

Palabras clave: Atención a la diversidad, adaptación curricular, acción tutorial en grupo, orientación.

\section{ABSTRACT}

The development of the LOGSE (1990) within the fied of «Attention to the diversity», at the highschools (IES) in the Valencian Autonomous Region has its reference in the framework of the «Orden de Atención a la Diversidad» (regulation for the attention to the diversity) from june $29^{\text {th }}, 1999$. This

* Doctor en CCEE por la UNED, Diplomado en EGB (Lengua Española e Idiomas). Profesor de Educación Secundaria en la especialidad de Psicología y Pedagogía, en la actualidad Jefe del Departamento de Orientación del Instituto de Educación Secundaria Matilde Salvador de Castellón de la Plana. Líneas de investigación: Atención a la diversidad en la Reforma Educativa. La diversidad en Educación Secundaria. Medidas específicas en distintas comunidades autónomas. 
regulation lays down the procedure planning to deal with the idea of «attention to the diversity» of the alumni, with both general and specific measures.

Within this article we try to do a detailed analysis and assessment of what it has been to set off the most especific meausure of attention to the diversity in our school, known as «programa de adaptación curricular en grupo» (adapted curriculum for the whole group program) and ant hte same time to draw a series of conclusions that have arisen from the experience with the students, teachers, results, orientation and support servicies, curriculum, syllabus, materials and resources and its incidence in our school.

Key words: Attention to the diversity, adapted curriculum, group program, training

\section{Introducción}

El Programa de Adaptación Curricular en Grupo es una medida excepcional de atención a la diversidad, dirigida al alumnado del segundo ciclo de la Educación Secundaria Obligatoria menor de 16 años que presenta serias dificultades de adaptación a la actividad escolar, que manifiesta graves desajustes de comportamiento en el ámbito escolar; que acumula un retraso escolar que hace muy difícil su desarrollo educativo en el grupo ordinario; que corre un grave riesgo de abandono del sistema escolar.

Dado su carácter excepcional, solo es necesaria la aplicación de esta medida, cuando se valora que no es suficiente la adopción de ninguna de las otras medidas de atención a la diversidad (de carácter general o específicas) a las cuales hace referencia la Orden de atención a la diversidad de la Consejería de Cultura, Educación y Ciencia de la Generalidad Valenciana. (Orden 19 de junio 1999. DOGV 29-6-99).

La aplicación de esta medida requiere una especial prudencia ya que supone para el alumno cursar un currículum muy diferenciado del ordinario. La unidad curricular fundamental del programa es el ámbito, entendido como un medio para integrar los aprendizajes básicos y fundamentales de las diversas áreas, de manera global.

El programa se estructura en tres grandes ámbitos: el Lingüístico y Social, el Científico, y el Tecnológico Plástico. Se pretende, de esta manera, que el alumnado pueda tener un seguimiento muy personalizado y pueda adquirir así los niveles de socialización, madurez y convivencia en los que presenta importantes carencias. Se trata de propiciar el desarrollo de todas las capacidades que se recogen en los objetivos generales de manera que el alumno pueda obtener el título de Graduado en Educación Secundaria siguiendo un programa de Diversificación Curricular, o en el caso en que esto no sea posible, pueda incorporarse a un Programa de Garantía Social.

La Orden de Atención a la Diversidad, especifica todos los pasos necesarios para poner en marcha estos programas y el proceso de incorporación del alumnado.

En definitiva se trata pues de:

a) Una medida de atención a la diversidad para favorecer la integración del alumnado del centro.

b) Desarrollar un currículo que contribuya a la obtención de los niveles adecuados de socialización, convivencia y madurez, al mismo tiempo que busca la recuperación de los déficits de aprendizaje. 
c) Desarrollar una metodología y contenidos adaptados a las necesidades de los alumnos.

d) Facilitar la incorporación a un Programa de Diversificación Curricular o a un Programa de Garantía Social.

e) Un programa dirigido al alumnado que presenta serias dificultades de adaptación a la actividad escolar, que acumula un retraso escolar significativo, que manifiesta deficiencias en la convivencia y graves problemas de comportamiento en el ámbito escolar y que sea menor de 16 años.

f) Programa propuesto por el profesorado del grupo correspondiente previa evaluación psicopedagógica, con autorización de los padres o tutores legales y con informe favorable de la Inspección Educativa.

\section{Contexto de la experiencia}

\section{El centro}

El I.E.S. Matilde Salvador es el primer centro que se construyó en Castellón siguiendo las directrices de la L.O.G.S.E. en cuestión de espacios, talleres, laboratorios, etc. La actividad académica se divide en tres turnos de mañana, vespertino y nocturno, y abarca el $1^{\mathrm{er}}$ y $2^{\circ}$ ciclo de la E.S.O., el Bachillerato L.O.G.S.E. completo (Tecnológico y Científico), grupos residuales de la antigua Formación Profesional, Programas de Garantía Social, y Ciclos Formativos de grado medio y superior de las familias Sanitaria y Construcción y Obra Civil.

Está ubicado en el extrarradio de la ciudad y recibe su alumnado de E.S.O. de los colegios de la zona, con familias de clase media y de un centro de acción educativa singular situado en un barrio marginal de las afueras de la ciudad.

\section{Necesidad del Programa}

Nuestro centro decidió adscribirse al P.A.C.G. puesto que necesitábamos dar soluciones a un grupo de alumnos que destacaban del resto de compañeros. Estos alumnos presentaban las siguientes características: Nivel académico que difícilmente superaba la educación Primaria. Ambiente familiar con problemas. Excesiva permisividad. Fuerte marginación social junto a un desinterés tanto personal como familiar.

Dadas estas circunstancias se solicitó la creación de un P.A.C.G. partiendo de dos premisas básicas:

- Número de alumnos a incorporar al grupo pequeño: 8-12 alumnos, abierto a nuevas incorporaciones.

- Objetivo triple:

- Personal: Desarrollo de la autoestima y socialización

- Académico: Posibilidad de incorporación a un PDC o un PGS

- Profesional: Preparación para la inserción en la vida laboral 


\section{Selección del alumnado}

Si leemos la orden de la atención a la diversidad en educación secundaria obligatoria, el perfil de estos alumnos viene determinado claramente (artículo 32):

- Retraso escolar manifiesto acumulado

- Problemas de adaptación al trabajo en el aula.

- Desajustes de comportamiento que dificultan el desarrollo de las clases.

- Alumnos en grave riesgo de abandono del sistema escolar.

\section{Procedimiento para la selección de la alumnado}

1. En nuestro centro, son alumnos que ya han repetido segundo curso de la educación secundaria y que a través de las diferentes juntas de evaluación se van seleccionando, para llegar al final del curso y en la junta de evaluación final establecer la propuesta definitiva.

2. Son chicos y chicas que no dejan dar clase, o no asisten a ellas, y que hacen muy difícil que se pueda continuar su desarrollo educativo en un grupo ordinario.

3. Tras acuerdo de la junta de evaluación se convoca a los padres y se les explica claramente su posibilidad de incorporar ese a este grupo de adaptación o continuar en un tercero ordinario.

4. También se ha incluido en el grupo algún alumno a petición directa del servicio de inspección.

\section{Problemática que se presenta a la hora de iniciar el grupo}

1. Cierta oposición de algunos profesores a esta medida, por considerar que se etiquetan a los alumnos.

2. Cierta reticencia por parte de alguna familia por aceptar que su hijo formara parte de este grupo aunque lo veía conveniente, sobre todo por el qué dirán.

3. Decidirnos a primar para acceder al grupo la conducta disruptiva antes que el retraso escolar.

En el artículo 34 del capítulo noveno de la orden de atención la diversidad se determinan los pasos a seguir para la incorporación de un alumno o alumna al programa:

a) Hemos seguido la propuesta razonada (por acuerdo de junta de evaluación final de $2^{\circ}$ nivel de ESO).

b) El departamento orientación no ha realizado la evaluación psicopedagógica puesto que ya conocíamos a los alumnos a lo largo de tres cursos (los dos ordinarios de primer ciclo y el de repetición de segundo, y teníamos los informes individuales de cada profesor de final de cada curso, y los informes globalizados de los profesores tutores de los tres años), si bien si que se ha oído al alumno y se les explicó claramente cómo iba a ser el grupo, la metodología a seguir y la finalidad, así como a los padres mediante entrevistas individuales y acuerdo manifiesto por escrito de incorporación al grupo. 
c) La decisión definitiva sobre la incorporación se tomó en sesiones a las que asistieron el tutor o tutora o los profesores de ámbitos junto con la jefatura de estudios y el jefe del departamento de orientación y los padres.

d) La dirección del centro autorizó la incorporación de los alumnos al programa. La inspección no informo ni favorable ni desfavorablemente pero sí hizo un seguimiento del proceso de incorporación

c) El proceso estuvo finalizado al inicio del curso académico 1999-2000, para ello no se incluyó a los alumnos en los listados iniciales de los grupos ordinarios de tercero.

\section{Selección del profesorado de ámbito}

Según el artículo 37 de la orden, el director del centro es el que designará al profesorado a propuesta de los departamentos didácticos.

Nos planteamos una doble cuestión:

a) Seleccionan primando la preparación y formación.

b) Seleccionar según las sensibilidad al problema.

En nuestro caso se pensó que era mejor la segunda opción, porque la preparación se van obteniendo a través de la experiencia y para trabajar con estos alumnos hace falta mucha sensibilidad, paciencia, motivación, etc. Por eso los profesores son voluntarios.

¿Qué está ocurriendo con los profesores que atienden a estos alumnos?

- Acusan de cierto desgaste personal.

- Sus cambios de humor son frecuentes.

- Sufren algunas experiencias personales desagradables.

- Se cuestionan algunas veces su manera de trabajar.

\section{Propuestas educativas orientadoras para el trabajo}

- Partir de la realidad. Es decir, ver las necesidades que tiene el grupo. Por eso el desarrollo del programa básico de los diferentes ámbitos se va construyendo a medida que va avanzando el curso.

- Generalmente se atiende a:

a) Necesidades cognitivas, (grandes lagunas de aprendizaje en áreas instrumentales).

b) Necesidades afectivas, (burlas, falta de respeto, baja autoestima, amonestaciones continuas...)

c) Desajustes emocionales, (frialdad en el trato, mentiras, agresividad...)

d) Necesidad de estar motivados, (hacer algo que entienden les motiva).

e) Necesidad de hacer un trabajo simulado, lo más posible al real, no como trabajo de aula.

f) Necesidad de tener conductas preferenciales para aprender.

Punto de partida en la experiencia: lograr que vengan a gusto y con ganas. 


\section{¿Qué supone esto?}

- Prescindir de los currículos oficiales. Los que aparecen en la orden (anexo XII) sirven de poco, sólo como punto de partida, hay que adaptarlos a cada centro. Hay que atender más a la capacidad espacial y partir siempre de cosas prácticas que le sirvan para la vida real.

- Intentar trabajar por el método de proyectos.

- Dificultades a la hora de evaluar a los alumnos.

- Solucionar los problemas a medida que vayan apareciendo.

Conclusión: el currículo debe adaptarse a las necesidades de cada tipo de alumnos.

\section{Principios importantes a tener en cuenta}

La experiencia nos dice que hay que ir de lo básico a lo general y de lo afectivo a lo cognitivo, ya que son alumnos con grandes carencias afectivas y con una necesidad urgente: ser tratados como personas, escucharles, antes que llenarlos de conocimientos, antes que darles clase.

Nuestra propuesta sería:

1. Potenciar el ámbito tutorial: ampliación de horas, contenidos, dinámica de grupo, habilidades sociales, adquisición de pauta de diálogo...

2. Añadir un ámbito de iniciación profesional: donde el alumno vea una finalidad clara de salida al mundo laboral. Con ello se conseguiría aumentar sus expectativas y autoestima.

\section{Repercusiones de la experiencia que nos ha supuesto esta manera de atender a la diversidad}

\section{Desde el punto de vista legal}

- Concienciación de que somos educadores antes que instructores, es decir, asumir la función tutorial como parte de la función docente.

- Que la atención a la diversidad no es vocacional, es por ley.

- Cambio de mentalidad didáctica del profesorado (la LOGSE ya lo presupone, pero en este programa se hace más patente).

\section{Desde el punto de vista académico}

- Plantea un problema profesional serio: falta experiencia, de propuestas, de medios materiales, de condiciones de trabajo

- Supone más trabajo para el docente: seminarios de formación y reuniones frecuentes. 


\section{Desde el punto de vista social}

- No son sólo alumnos de clase baja sino también de clase media.

- Muchos padres no saben qué hacer con ellos.

- El problema se acentúa por la obligatoriedad hasta los dieciséis años.

\section{Desde el punto de vista psicológico}

- Los alumnos que nos llegan generalmente son muy inmaduros porque:

- Han perdido todas los expectativas académicas (interés).

- Todo lo que suene a aula lo rechazan.

- No quieren hacer nada

- No conocen las metodologías de trabajo.

- Son adolescentes y se ven mayores y no quieren hacer caso a nadie.

- Necesitan sentirse «alguien».

\section{Conclusiones de la experiencia. Curso escolar 1999-2000 y análisis de desarrollo en el curso 2000-01}

Consideraciones derivadas de la experiencia en los diferentes aspectos:

\section{En relación con el alumnado:}

- Evaluación psicopedagógica especial de tipo cualitativo y cuantitativo.

- Posibilidad de PACG de dos años de duración

- Los alumnos que acaban con 15 años, posibilidad de incorporación a programa especial, con finalidad de inserción laboral.

- Posibilidad de paso a curso preparatorio de acceso a CFGM, (programa académico específico).

\section{En relación a los resultados:}

- Se observa una mejora en la actitud de los alumnos.

- Respecto a los currículos se observa una mejora en los aspectos procedimentales y actitudinales y poco en los conceptuales.

- Necesidad de aplicaciones prácticas en el programa y en el trabajo del día a día.

\section{Profesorado y servicios de orientación y apoyo:}

- Necesidad de mayor coordinación entre los ámbitos curriculares, en torno a proyectos globalizadores e interdisciplinares, con contenidos procedimentales y siguiendo los intereses del alumnado.

- El profesorado de los ámbitos ha de reunir un conjunto de habilidades docentes que faciliten la convivencia y el orden en la clase y al mismo tiempo que tenga la capacidad de crear un clima estimulante de iniciativas y motivador. 
- Necesidad de conocer de antemano el material humano con el que van a trabajar y con apoyo del equipo directivo y profesorado del centro.

- Profundizar en una metodología didáctica de carácter globalizadora e interdisciplinar en donde se dé la mayor coordinación posible.

\section{Currículo, programación, materiales y recursos:}

- La falta de experiencia y la necesidad de facilitar resultados a corto plazo ha forzado al profesorado a una planificación basada en actividades de corta duración en muchos casos.

- Necesidad de replantear un currículo pensado y acordado previamente para afrontar las variables de comportamiento y rendimiento, pero con carácter abierto $\mathrm{y}$ flexible.

- Un modelo de secuencia didáctica diaria de trabajo con alumnos de este grupo sería:

- Actividad tutorial de inicio (plan de actividades del día, clima y actitud)

- Desarrollo del programa de ámbito.

- Taller y actividades procedimentales.

- Autoevaluación del grupo y de los objetivos del día.

- Respecto a la acción orientadora y tutorial se sugiere:

- Tutoría matinal con actividades de inicio que motiven y orienten en trabajo diario.

- Tutoría temática específica para el tratamiento de referentes que mejoren el desarrollo personal del alumnado.

- Tutoría de proceso en donde cada profesor oriente a nivel curricular y corrija y regule los hechos puntuales que exijan intervención oportuna.

- Necesidad de un aula con el perfil de aula-taller, bien dotada de recursos didácticos e informáticos.

\section{Respecto a la incidencia en el centro:}

- La experiencia ha resultado positiva, puesto que se favorece el clima de convivencia y trabajo en el desarrollo de las clases con los grupos ordinarios.

- El equipo directivo y el profesorado del centro debe colaborar en el buen desarrollo del programa implicándose al máximo en el mismo, sobre todo cuando los alumnos se encuentran en espacios comunes, aulas ordinarias o actividades extraescolares. 